\title{
Effect of Molecular Weight on the Feature Size in Organic Ice Resists
}

\author{
Elsukova, Anna; Han, Anpan; Zhao, Ding; Beleggia, Marco
}

\section{Published in:}

Nano Letters

Link to article, DOI:

10.1021/acs.nanolett.8b03130

Publication date:

2018

Document Version

Peer reviewed version

Link back to DTU Orbit

\section{Citation (APA):}

Elsukova, A., Han, A., Zhao, D., \& Beleggia, M. (2018). Effect of Molecular Weight on the Feature Size in Organic Ice Resists. Nano Letters, 18(12), 7576-7582. https://doi.org/10.1021/acs.nanolett.8b03130

\section{General rights}

Copyright and moral rights for the publications made accessible in the public portal are retained by the authors and/or other copyright owners and it is a condition of accessing publications that users recognise and abide by the legal requirements associated with these rights.

- Users may download and print one copy of any publication from the public portal for the purpose of private study or research.

- You may not further distribute the material or use it for any profit-making activity or commercial gain

- You may freely distribute the URL identifying the publication in the public portal 


\title{
Effect of molecular weight on the feature size
}

\section{in organic ice resists}

\author{
Anna Elsukova ${ }^{1}$, Anpan $\mathrm{Han}^{2}$, Ding Zhao ${ }^{1}$, and Marco Beleggia ${ }^{1 *}$ \\ ${ }^{1}$ DTU Danchip/Cen, Technical University of Denmark, 2800 Kongens Lyngby, Denmark \\ ${ }^{2}$ DTU Mechanical Engineering, Technical University of Denmark, 2800 Kongens Lyngby, Denmark
}

*marco.beleggia@cen.dtu.dk

\section{Table of Contents Graphic}

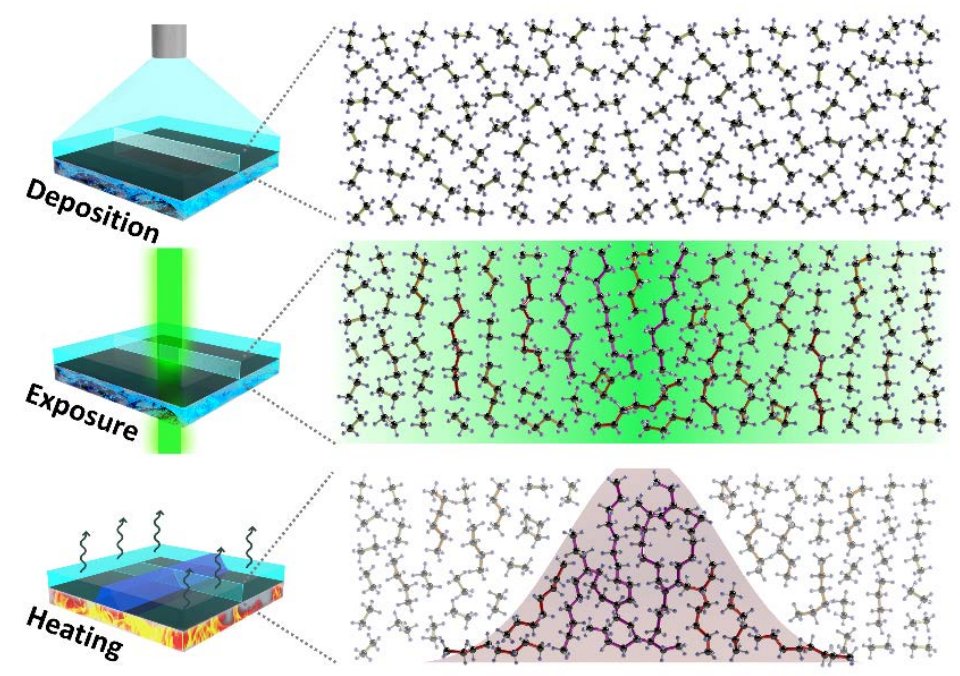

\section{Abstract}

The feature size of patterns obtained by electron beam lithography (EBL) depends critically on resist properties, beam parameters, development process, and instrument limitations. Frozen layers of simple organic molecules such as n-alkanes behave as negative tone resists for EBL. With the unique advantage of an in-situ thermal treatment replacing chemical development, the entire lithographic process can be performed within a single instrument, thus removing the influence of chemical developers on the feature 
size. By using an environmental transmission electron microscope, we can also minimize the influence of instrumental limitations and explore the fundamental link between resist characteristics and feature size. Our results reveal that the onset dose of organic ice resists correlates with the inverse molecular weight, and that in the thermal development the role of change in solubility of polymers is mirrored in a shift in the solid/vapour critical temperature of organic ices. With a $0.4 \mathrm{pA}$ beam current we obtained $4.5 \mathrm{~nm}, 5.5 \mathrm{~nm}$, $8.5 \mathrm{~nm}$, lines with frozen octane, undecane, and tetradecane, respectively, consistent with the predictions of a model we developed that links beam profile and feature size. The knowledge acquired on the response of small organic molecules to electron irradiation, combined with the flexibility and operational advantages of using them as qualified EBL resists, provide us with new opportunities for the design and production of nanodevices, and broadens the reach of EBL especially towards biological applications.

\section{Keywords}

E-beam lithography, organic ice resist, condensed organic molecules, crosslinking, transmission electron microscopy, exposure mechanism

During the last 50 years electron beam lithography (EBL) has advanced significantly, and can now deliver structures that are just a few nanometers in size. EBL is used in many research areas such as advanced electronics, ${ }^{1}$ quantum computing ${ }^{2}$, nanophotonics ${ }^{3}, 2 \mathrm{D}$ material devices ${ }^{4}$, optomechanical ${ }^{5}$ and nanofluidics systems ${ }^{6}$. EBL is also expanding into the patterning of biomolecules ${ }^{7,8}$, with potential applications ranging from tissue engineering, to drug delivery, to fabrication of biosensors and bio-interfaces for diagnostics and cell stimulation ${ }^{9,10}$. An emerging trend in many application fields is three-dimensional (3D) device fabrication ${ }^{11}$. To satisfy the broad range of practical demands, new resist materials ${ }^{12}$ and approaches to 
electron-beam (e-beam) based nanopatterning are being considered ${ }^{13,14}$. In this perspective, we have recently introduced the concept of Organic Ice Resists (OIR $)^{15}$. Instead of conventional spin-coating and chemical development, we first freeze simple organic molecules at cryogenic temperature (Figure 1a,b), and then expose them to a focused electron beam (Figure 1c), leading to crosslinking of the molecules (Figure 1d). After thermal development by heating the sample, light molecules will leave the substrate while heavily crosslinked molecules within the exposure area are solidified (Figure 1e,f). Therefore, these organic materials can act as negative-tone resists in EBL. Repeated condensation-exposure cycles yielding 3D layered structures can be efficiently fabricated in a single instrument ${ }^{16,17}$. The use of biocompatible organic materials and the chemical-free development process could potentially make OIR-based lithography (OIRL) the most convenient method for nanofabrication of bio-devices. Finally, OIRL handles easily substrates with complex morphologies (for example, a thin microporous $\mathrm{Si}_{3} \mathrm{~N}_{4}$ membrane ${ }^{15}$ ). Since the resist material condenses from vapor, it can be uniformly deposited on porous, grooved and curved surfaces in a single step. This is particularly important for nanofluidic applications, and is currently beyond the reach of conventional EBL or nanoimprint patterning protocols ${ }^{18}$.

In this work, we take the first steps towards a deeper understanding of the principles behind e-beam exposure of organic ices, and of the role played by the thermal development. Acquiring knowledge on the radiation chemistry processes driving the OIR exposure mechanism, and on how it relates to the characteristics of the patterns, is crucial for identifying the optimal organic material, lithographic protocol and control over the structures' functionality in view of specific applications. The fundamental question we address here is what controls the feature size achievable in OIRL, and how it relates to the initial molecular weight of the chosen organic compound. For this purpose, we have chosen to pattern the smallest and the simplest organic molecules with suitable freezing points: linear hydrocarbons octane $\mathrm{C}_{8} \mathrm{H}_{18}$, undecane $\mathrm{C}_{11} \mathrm{H}_{24}$, and tetradecane $\mathrm{C}_{14} \mathrm{H}_{30}$ (Figure $1 \mathrm{~g}$ ). These molecules have a simple linear structure with only $\mathrm{C}-\mathrm{C}$ and $\mathrm{C}-\mathrm{H}$ bonds. Therefore, we expect their molecular weight to be the key parameter determining the patterned feature size. 
To minimize the influence of instrumental limitations on the feature size, for example a broad beam spot and optics instabilities, we used an environmental transmission electron microscope (ETEM) ${ }^{19}$ equipped with a gas cell and a cryo-holder to condense OIR on a 5-nm thick $\mathrm{Si}_{3} \mathrm{~N}_{4}$ membrane, and operated at $80 \mathrm{keV}$. Images of the resulting OIR patterns were obtained in-situ, immediately after the substrate was heated. The microscope optics allowed us to change the e-beam current continuously and independently from the beam spot size. The spot size in a TEM operated in scanning mode is controlled by the C2 (second condenser) aperture and by the excitation of the objective lens, situated below. The beam current is set by adjusting the strength of the lenses above the $\mathrm{C} 2$ aperture, which disperses and condenses the illumination on it, thereby changing the number of electrons passing through without affecting the spot size. Therefore, we could set a small beam spot $(\sim 1 \mathrm{~nm})$ and study the patterned linewidth as function of dose. The beam current was measured using a calibrated CCD camera by counting the total number of electrons detected over a given exposure time. This is equivalent to measuring the current by a Faraday cup. The experimental setup and procedure are described in detail in the Supporting Information. Figure $1 \mathrm{~h}$ shows a simplified diagram of the phase transitions occurring during the OIRL process. First, an organic vapor is condensed into an amorphous ice layer at temperature $T_{L N 2}$. Then, electron beam irradiation initiates the breakdown of chemical bonds of the original molecules, leading to their subsequent crosslinking. After crosslinking, the irradiated region has a larger average molecular weight and thus higher sublimation temperature $T_{\text {sub. After heating to }}$ temperature $T_{h}$, all molecules with $T_{\text {sub }}>T_{h}$ remain on the substrate. We do not observe any liquid phase present after heating. However, it is possible that they could form and evolve during heating (not shown in Figure $1 \mathrm{~h}$ ). The final products of the OIRL process are hydrogen containing carbonaceous structures, which are non-volatile, chemically inert and very stable at ambient conditions ${ }^{15}$. 

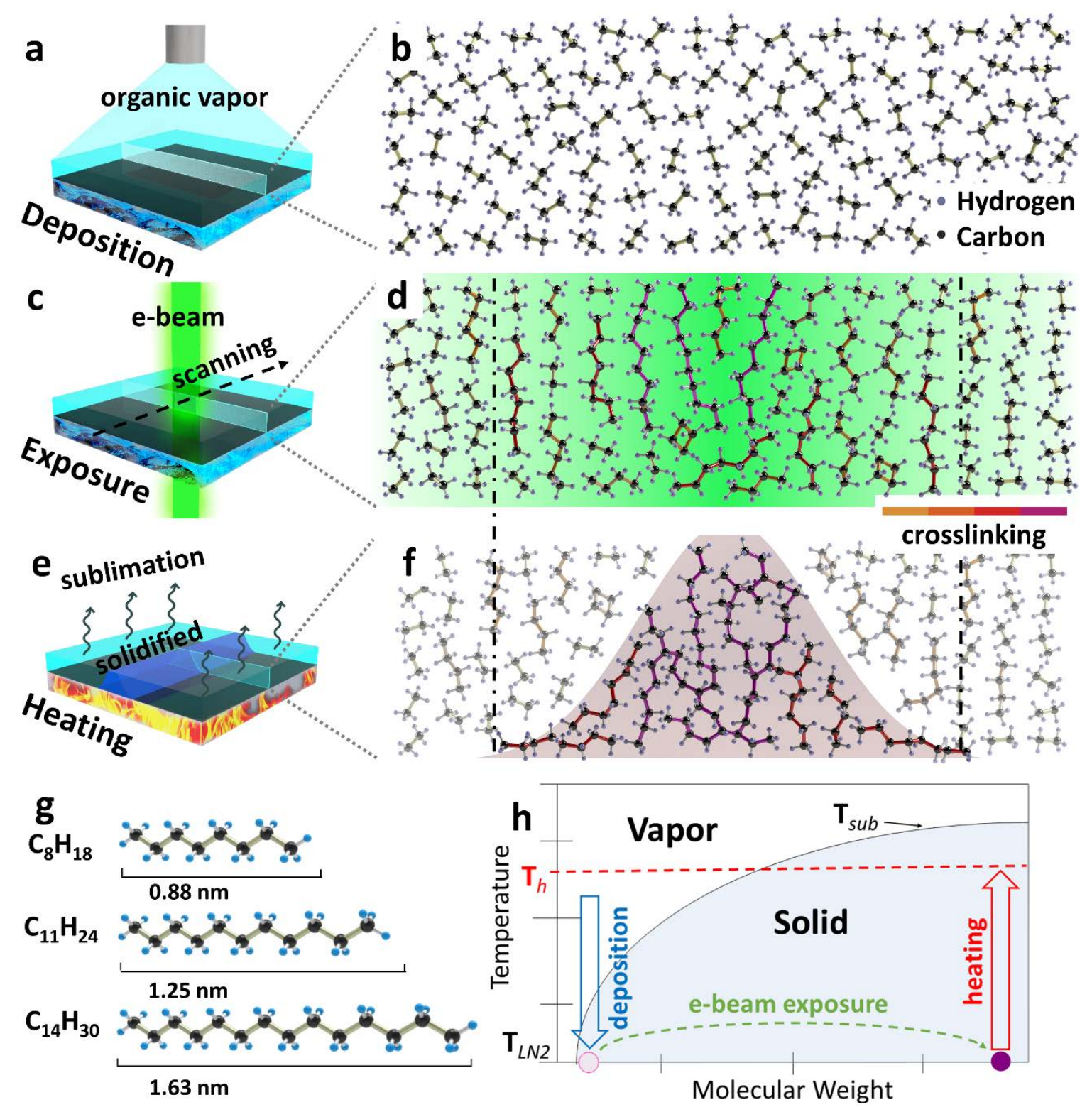

Figure 1. Organic ice resists lithography and interactions between electron beam and ice resists. (a) A layer of deposited amorphous organic ice (light blue) on a cold substrate surface and (b) an illustration of its cross sectional view. (c) Exposing the ice layer to a focused electron beam (green) with a Gaussian profile leads to (d) crosslinking of the molecules. The electron beam current density decreases gradually from the center (dark green) out to the periphery (light green), and the degree of crosslinking depends on the received dose. The dash-dot lines represent the borders within which organic ice receives enough dose to form a non-volatile product at $T_{h}$. (e) The solidified line pattern (dark blue) that remains within the scanned area after heating the sample to temperature $T_{h}(40$ $\left.{ }^{\circ} \mathrm{C}\right) .(\mathrm{f})$ The approximate cross-sectional profile of the line pattern (shaded area). The pattern thickness is proportional to the number of molecules crosslinked enough to become non-volatile at $T_{h}$. $(\mathrm{g})$ Molecular dimensions of the linear hydrocarbons octane $\mathrm{C}_{8} \mathrm{H}_{18}$, undecane $\mathrm{C}_{11} \mathrm{H}_{24}$, and tetradecane $\mathrm{C}_{14} \mathrm{H}_{30}$. (h) Simplified phase diagram underpinning the OIRL process. 
After testing and adjusting the deposition parameters, we deposited a thin (12-15 nm) layer of ice and found the patterning conditions that delivered the smallest linewidths. Figure 2 shows parallel lines patterned on octane (a-c), undecane (d-f) and tetradecane (g-i) using beam currents $l_{o L}$ of $0.4 \mathrm{pA}(\mathrm{a}, \mathrm{d}, \mathrm{g}), 0.66 \mathrm{pA}(\mathrm{b}, \mathrm{e}, \mathrm{h})$ and $1.6 \mathrm{pA}(\mathrm{c}, \mathrm{f}, \mathrm{g})$, and dwell time per dot $\tau_{L}=10 \mathrm{~ms}$. The line dose is defined as $D=I_{O L} \tau_{L} / \Delta x_{L}$, where $\Delta x_{L}=L / N$, $L=360 \mathrm{~nm}$ is the length of the line, $N=100$ is the number of dots. We carefully estimated the linewidths from bright-field TEM images by fitting the out-of-focus intensity profile with an analytical model for a Gaussian weak phase object. Details on linewidth estimation from TEM images are discussed in the Supporting Information. In our previous work ${ }^{15}$, we characterized the resulting patterns with Energy Dispersive X-ray (EDX) and Electron Energy-Loss (EELS) spectroscopies. EDX showed only presence of carbon, and the EELS carbon K-edge had the typical shape of hydrogenated carbon in an amorphous environment. Hydrogenated carbon produces a weak contrast in TEM and is prone to beam damage, which leads to carbon sputtering and thinning of structures. To minimize damage and increase the contrast, we imaged the structure under-focus at low-dose.

The experimental data show two trends: 1) the linewidth decreases with the beam current (line dose) (Figure $2 \mathrm{j}$ ), and 2) for a fixed beam current (line dose), the linewidth decreases with the decreasing initial molecular weight of the precursor (Figure $2 \mathrm{k}$ ). The smallest linewidth of $4.5 \mathrm{~nm}$ was achieved on octane using a beam current of $0.4 \mathrm{pA}$ (Figure 2a). 

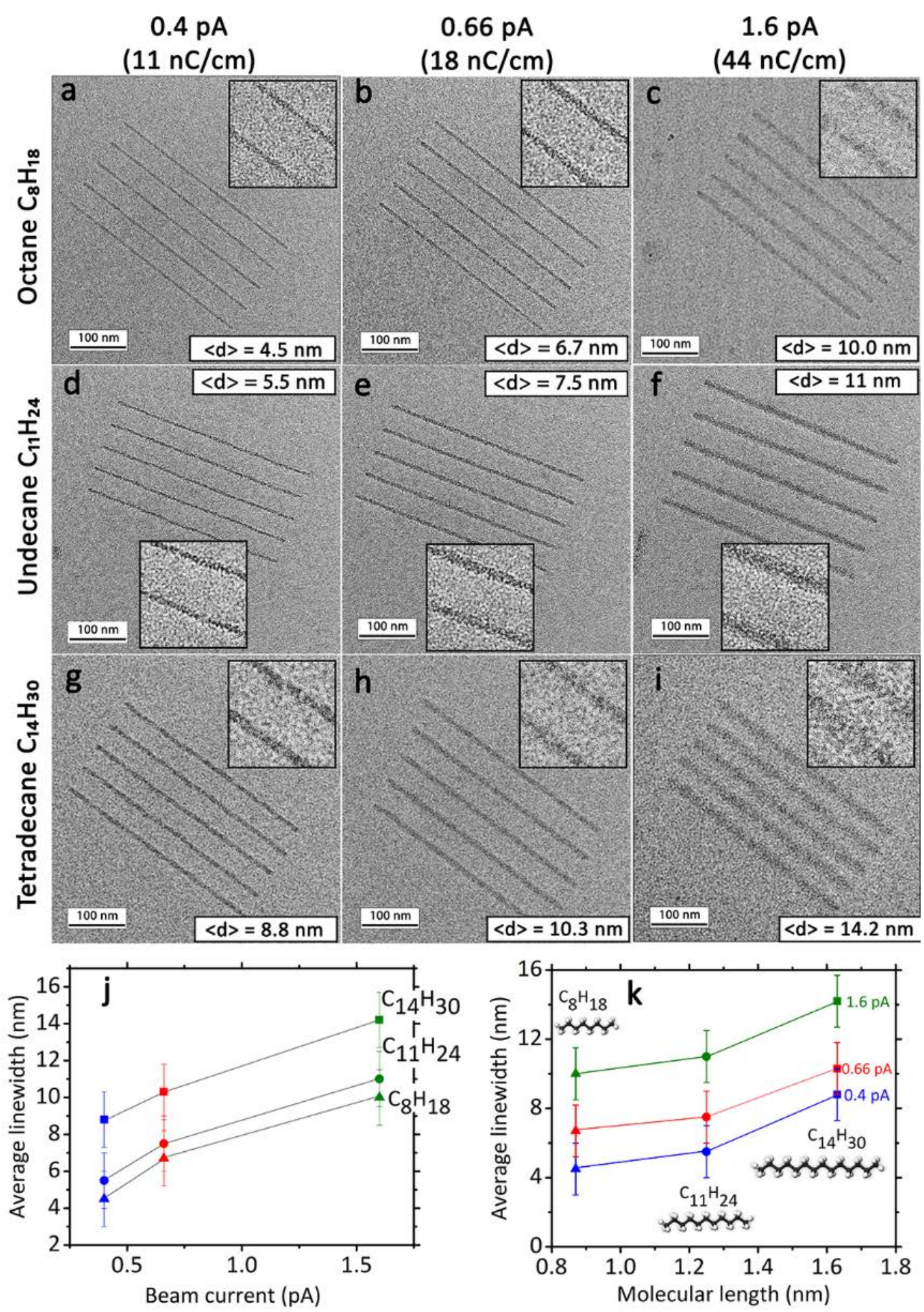

Figure 2. Parallel lines patterned on octane (a)-(c), undecane (d)-(f) and tetradecane (g)-(i) OIRs using beam currents 0.4 pA (a, d, g), $0.66 \mathrm{pA}(\mathrm{b}, \mathrm{e}, \mathrm{h})$ and $1.6 \mathrm{pA}(\mathrm{c}, \mathrm{f}, \mathrm{g})$. Patterning parameters: line length $L=360 \mathrm{~nm}$, dwell time per dot $\tau_{L}=10 \mathrm{~ms}$, number of dots $N=100$. Line dose is defined as $D=I_{O L} \tau_{L} / \Delta x_{L}$, where $I_{L L}$ is the beam current, $\Delta x_{L}=L / N$. Bottom row: Average linewidths of the patterned structures plotted as a function of $(\mathrm{j})$ beam current and $(\mathrm{k})$ OIR precursor molecular length. 

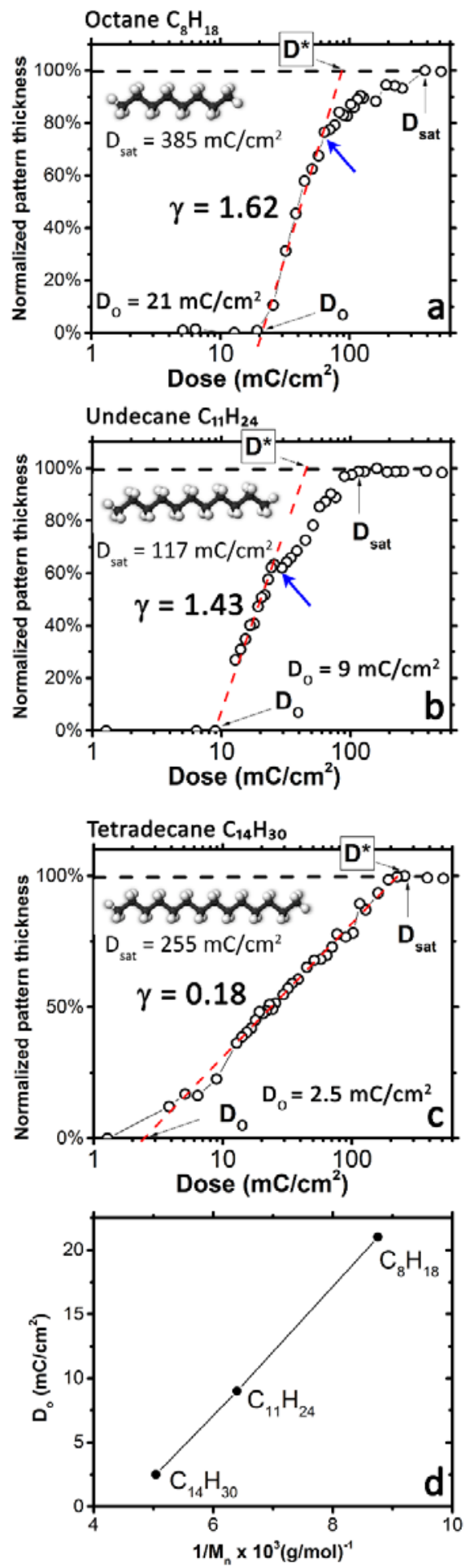

Figure 3. Contrast curves for (a) octane, (b) undecane and (c) tetradecane OIRs. (d) Onset dose $\left(D_{0}\right)$ dependence on OIR molecular weight. The pattern thickness is normalized to the thickness of a highly exposed area. Contrast $\gamma=1 / \log _{10}\left(D^{*} / D_{0}\right)$, where $D_{0}$ and $D^{*}$ are determined by extrapolating the linear portion of the contrast curve to 0 and $100 \%$, respectively. $D_{\text {sat }}$ represents a saturation dose. Blue arrows in (a, b) highlight a visible change of slope in the contrast curve. 
To gain insight into the exposure mechanism of organic ices, we acquired a set of contrast curves (Figure 3ac) for the three compounds examined. We patterned $500 \times 500 \mathrm{~nm}^{2}$ squares with a beam current of $3 \mathrm{pA}$. Patterning was carried out by obtaining a $1024 \times 1024$ pixels STEM image in the ETEM. The dose was controlled by changing the dwell time per pixel in the range $0.5-400 \mu \mathrm{s}$. The curves shown in Figure 3 are plotted as a function of area dose defined as $D=I_{O A} \tau_{\AA} /\left(\Delta x_{A}\right)^{2}$, where $I_{O A}$ is the beam current, $\tau_{\mathrm{A}}$ is the dwell time per dot and $\Delta x_{\mathrm{A}}=500 / 1024 \mathrm{~nm}=0.49 \mathrm{~nm}$ is the pixel size. The thickness of the exposed square was estimated with the log-ratio method using electron energy-loss spectroscopy $(E E L S)^{20}$. The pattern thickness is normalized to the thickness of a highly exposed area, which is estimated to be $\sim 50 \mathrm{~nm}$ from collapsed features. The contrast curves are characterized by an onset dose $D_{0}$, below which no organic product remains after development, a saturation dose $D_{\text {sat }}$, after which the thickness of the pattern does not increase further, and a contrast, defined as $\gamma=1 / \log _{10}\left(D^{*} / D_{O}\right)$. $D_{O}$ and $D^{*}$ are determined by extrapolating the linear portion of the contrast curve to 0 and $100 \%$, respectively. The comparison between the measured contrast curves reveals a key finding: the onset dose decreases significantly with increasing molecular weight (Figure $3 \mathrm{~d}$ ), and follows an inverse proportionality trend observed for much heavier polymers ${ }^{21}$. The contrast also decreases with increasing molecular weight. It should be noted that the contrast of $\mathrm{C}_{8} \mathrm{H}_{18}$ and $\mathrm{C}_{11} \mathrm{H}_{24}$ ice resists are 1.62 and 1.43 , respectively, and that of $\mathrm{C}_{14} \mathrm{H}_{30}$ is only 0.18 , indicating that these linear hydrocarbons are low sensitivity resists, which may be unsuitable for dense patterns. The correlation of $D_{\text {sat }}$ with molecular weight is less obvious: it appears to decrease in undecane compared to octane, but then increases for tetradecane. The observed trends of onset dose, contrast and saturation dose for OIR are compared to polystyrene resists with different molecular weights ${ }^{22}$ in the Supporting Information (Figure S7), confirming that the trend in $D_{\text {sat }}$ is unusual.

In general, the linewidth and contrast curves obtained with negative EBL resists depend on many parameters such as resist material, exposure dose, beam size and energy, writing protocol and development process ${ }^{23}$. However, two general trends are observed ${ }^{22-24}$. First, high resolution resists require higher doses to achieve onset and saturation conditions. Second, with identical chemical structure and lithography protocol, resists 
with a lower molecular weight are expected to deliver smaller features. The resolution limit for individual features was studied on chain-structure polystyrene as a function of molecular weight ${ }^{21}$. The dot diameter decreased from 25 to $10 \mathrm{~nm}$ with molecular weight decreasing from 17000 to $800 \mathrm{~g} / \mathrm{mol}$.

The conventional EBL process is based on the solubility variation of the irradiated region due to the change in molecular weight ${ }^{23}$. In contrast, OIRL is based on the change in critical solid-(liquid)-vapor phase transition temperature $T_{c r}$. Figures $4 a, b$ illustrate schematically how an OIR contrast curve would be generated. The black lines in Figure 4a represent the molecular weight distribution in OIR before $\left(f_{0}(M)\right)$ and after $\left(f_{D 1}(M)\right.$, $\left.f_{D 2}(M), f_{D 3}(M)\right)$ e-beam exposure for doses $D 1, D 2, D 3 . M_{0}$ is the initial molecular weight of the organic precursor. The red curve in Figure 4a represents the expected dependency of $T_{c r}$ on molecular weight, based on the melting points data for linear hydrocarbons ${ }^{25} . M_{\text {crit }}$ marks the molecular weight above which the compound becomes non-volatile at temperature $T_{h}$. Exposing a hydrocarbon with initial molecular weight distribution $f_{0}(M)$ crosslinks the molecules and forms a new distribution $f_{D}(M)$. We expect the average molecular weight and the width of the distribution to increase with the dose. If the entire distribution $f_{D}(M)$ lies below a critical value $M_{c r i t}$, all molecules will leave the substrate after heating $\left(f_{D 1}(M)\right)$. This occurs at doses smaller than the onset dose $D_{0}$ (Figure $4 \mathrm{~b}$ ), where the OIR contrast curve is zero. Above $D_{0}$ and below $D_{\text {sat }}, M_{\text {crit }}$ lies somewhere within the distribution $\left(f_{D 2}(M)\right)$, and only the fraction of the molecules with $M>M_{\text {crit }}$ remains after development, producing the portion of the contrast curve in the range $D_{0}<D<D_{\text {sat }}$. Above the saturation dose $\left(D>D_{\text {sat }}\right)$ the entire distribution $f_{D}(M)$ lies above $M_{\text {crit, }}$ and all molecules remain after sublimation. This description implies that organic precursors with larger initial molecular weight require less crosslinking events, and therefore lower doses, to cross the $M_{\text {crit }}$ threshold and form a $T_{h}$-stable product. This behavior should manifest in lower onset and saturation doses (Figure $4 \mathrm{c})$. For smaller molecules $\left(M_{0}^{\prime}\right.$, Figure 4c), these doses are higher. Experimental contrast curves (Figure 3a-c) show that it is indeed the case for the onset doses. 
However, two features of the experimental contrast curves cannot be explained solely by the simple mechanism described above: the trend of saturation doses, and the change in slope observed for octane at $\sim 70 \mathrm{mC} / \mathrm{cm}^{2}$ and for undecane at $\sim 30 \mathrm{mC} / \mathrm{cm}^{2}$ (marked with blue arrows in Figure $3 a, b$ ). These observations suggest that the change in $T_{c r}$ from the exposure may not be uniquely defined by a change of molecular weight, and that some other factor may be in play that influences the shape of the contrast curves. Aside from molecular weight, the physical properties of organic compounds, including critical temperatures, depend on intermolecular forces, which in turn depend on molecular symmetry and architecture ${ }^{26,27}$. For example, linear n-octane has a melting point $T_{m}=-57{ }^{\circ} \mathrm{C}$. Its isomers 3-methylheptane and 2,5dymethylhexane have lower melting points $-122{ }^{\circ} \mathrm{C}$ and $-93{ }^{\circ} \mathrm{C}$, respectively. However, the octane isomer tetramethylbutane has a much higher melting point $T_{m}=98^{\circ} \mathrm{C}$. This suggests that instead of a single threshold $M_{c r i t}$, we deal with a distribution of critical molecular weights applicable to various types of topologies of crosslinked structures. Consequently, the fraction of molecules remaining after heating depends both on the increase in average molecular weight and on the change in molecular shape. Achieving a deeper understanding of molecular shape evolution with dose and its effect on contrast curves requires separate investigations involving Molecular Dynamics and Monte Carlo simulations.

However, our experimental observations clearly indicate that feature size and onset doses correlate with the initial precursor molecular weight. In order to understand better these correlations, we need to determine how the onset dose relates to the patterned linewidth. The characteristics of patterned features in the form of isolated dots or lines depend on the beam profile, especially on the beam tails where the dose rate (beam current density) drops. With a Gaussian beam, the onset dose determines the feature size as illustrated in Figure 5a. Away from the Gaussian center, the degree of crosslinking is not sufficient for the exposed ice to become non-volatile at heating temperature $T_{h}$. Precursors with higher onset doses $D_{O}$ will, therefore, result in smaller feature sizes. 

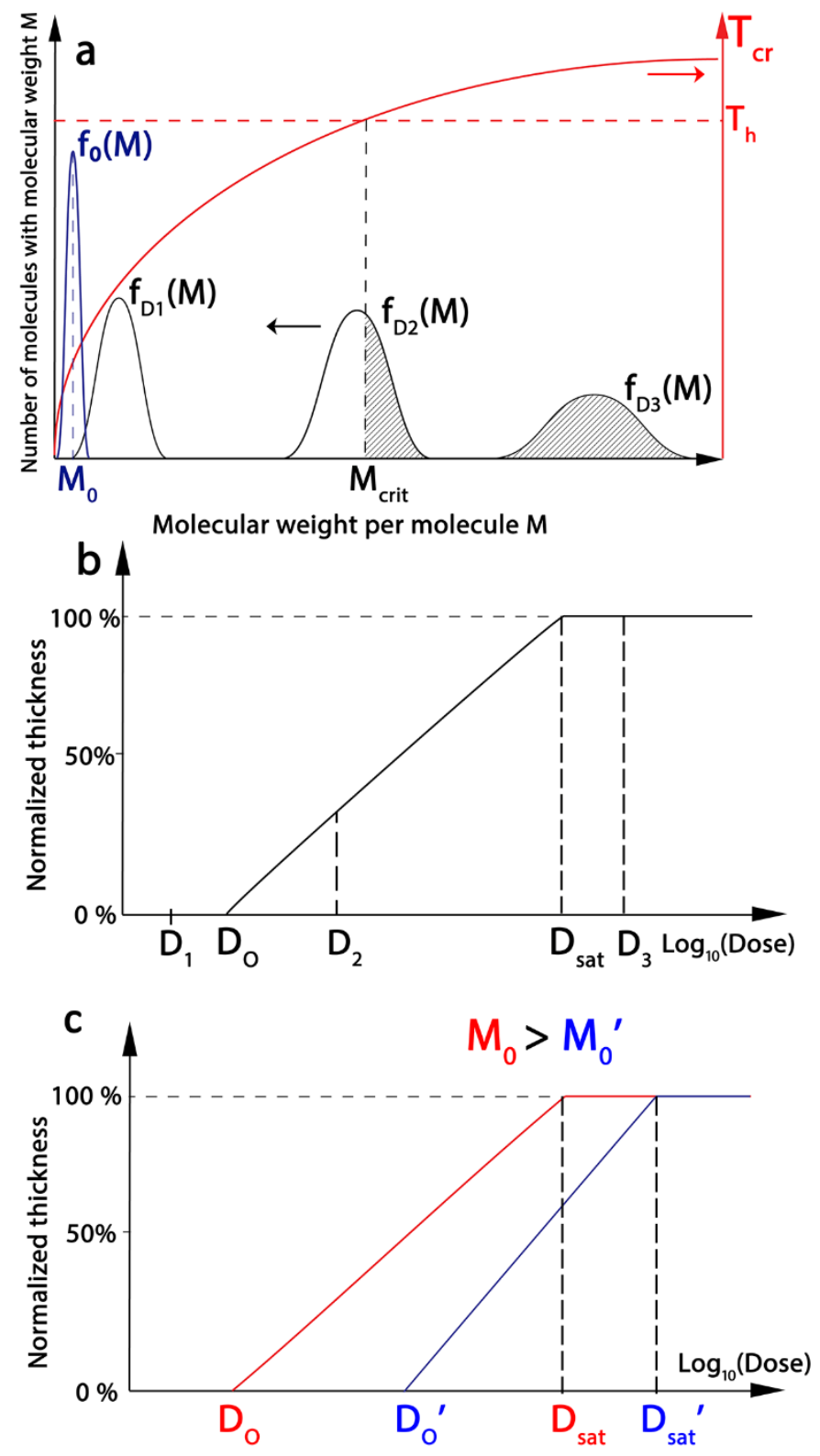

Figure 4. OIR contrast curve formation by e-beam exposure and thermal development. (a) Left $Y$ axis: molecular weight distribution in OIR before $\left(f_{0}(M)\right)$ and after $\left(f_{D 1}(M), f_{D 2}(M), f_{D 3}(M)\right)$ e-beam exposure for doses $D 1, D 2, D 3 . M_{0}$ is the initial molecular weight of organic precursor. Right $Y$ axis: critical temperature dependency on molecular weight. Molecular weight $M_{\text {crit }}$ defined as $T_{\text {sub }}\left(M_{\text {crit }}\right)=T_{h}$. (b) Corresponding contrast curve: the normalized pattern thickness at a particular dose $D$ depends on the shape of the molecular weight distribution $f_{\mathrm{D}}(M)$, and on how its position with respect to $M_{\text {crit. }}$ (c) Contrast curves for two precursors with different initial molecular weights $M_{0}$ and $M_{0}^{\prime}<M_{0}$. Organic ices with lower molecular weight require more crosslinking events to produce a nonvolatile product, therefore their contrast curves have higher values of onset $\left(D_{O}^{\prime}>D_{O}\right)$ and saturation doses $\left(D_{\text {sat }}{ }^{\prime}>D_{\text {sat }}\right)$. 
We consider a primary electron beam with a Gaussian profile, peak beam current density $j_{0}$ and beam radius $R_{\mathrm{p}}:$

$$
j_{p}(r)=j_{0} e^{-\frac{r^{2}}{2 R_{p}^{2}}}
$$

We expect crosslinking to occur mainly due to secondary electrons (SEs) for two reasons: i) we pattern a thin layer on a very thin substrate with $80 \mathrm{keV}$ electrons, making the influence of backscattered electrons negligible; ii) any relevant inelastic cross section from $80 \mathrm{keV}$ primaries is negligible with respect to that at the energy range $(<50 \mathrm{eV})$ of SEs, since the cross section drops with $1 / E^{28}$. Even if the $\sim 10000 \mathrm{x}$ drop in cross section may be partially offset if the effective yield of generated secondaries is below unity, the role of primaries in the exposure process may be safely neglected. Therefore, we assume that the electron flux driving the exposure process is

$$
j_{S E}(r)=Y j_{0} e^{-\frac{r^{2}}{2 R_{S}^{2}}}
$$

where $Y$ is an effective yield of generated SEs, representing the statistical number of SEs generated per primary, and $R_{S}$ is the Gaussian radius of the spatial distribution of SEs, which we roughly expect to be $R_{S} \approx$ $\sqrt{R_{p}^{2}+\lambda^{2}}$, where $\lambda$ is the inelastic mean free path of SEs at their mean energy. Therefore, the dose received by the ice over a time interval $\tau$ as a function of position $r=\sqrt{x^{2}+y^{2}}$ is

$$
D_{S E}(r)=Y j_{0} \tau e^{-\frac{r^{2}}{2 R_{S}^{2}}}
$$

When patterning a line (Figure 5b) or an area with overlapping beams we need to consider the contribution from the exposure of neighboring dots. In the assumption $\Delta x_{L} \leq R_{S}$ (significant overlap) the dot dose in a line $D_{L}$ can be estimated as: 


$$
\begin{gathered}
D_{S E_{L}}(y)=Y j_{0 L} \tau_{L}\left[\ldots+e^{-\frac{(-\Delta x)^{2}+y^{2}}{2 R_{S}^{2}}}+e^{-\frac{y^{2}}{2 R_{S}^{2}}}+e^{-\frac{(\Delta x)^{2}+y^{2}}{2 R_{S}^{2}}}+\cdots\right]=Y j_{0 L} \tau_{L} e^{-\frac{y^{2}}{2 R_{S}^{2}}}\left[\sum_{i=-\infty}^{+\infty} e^{-\frac{(i \Delta x)^{2}}{2 R_{S}^{2}}}\right] \approx \\
\approx Y j_{0 L} \tau_{L}\left(\frac{\sqrt{2 \pi} R_{S}}{\Delta x_{L}}\right) e^{-\frac{y^{2}}{2 R_{S}^{2}}}
\end{gathered}
$$

Following the same logic, dot dose in area is $D_{S E_{A}}=Y j_{0 A} \tau_{A}\left(\frac{\sqrt{2 \pi} R_{S}}{\Delta x_{A}}\right)^{2} \cdot j_{0 L}, \tau_{L}, j_{0 A}, \tau_{A}$ are primary beam current density, dwell time for line and area patterning, respectively. The patterned linewidth $d=2 y_{D O}$ (Figure 5b) can be found by solving the equation $D_{S E_{L}}\left(y_{D O}\right)=\left(D_{S E}\right)_{O}=\left(D_{S E_{A}}\right)_{O}$ :

$$
Y j_{0 L} \tau_{L}\left(\frac{\sqrt{2 \pi} R_{S}}{\Delta x_{L}}\right) e^{-\frac{y_{D O}^{2}}{2 R_{S}^{2}}}=Y\left(j_{0 A}\right)_{O}\left(\tau_{A}\right)_{O}\left(\frac{\sqrt{2 \pi} R_{S}}{\Delta x_{A O}}\right)^{2}
$$

Here $\left(j_{0 A}\right)_{O},\left(\tau_{A}\right)_{O}$ and $\Delta x_{A O}$ are area patterning parameters for the onset condition. As previously stated, the experimental onset dose $D_{O}$ is defined as $D_{O}=\frac{I_{0 A}\left(\tau_{A}\right)_{O}}{\Delta x_{A}{ }^{2}}=\frac{j_{0 A}\left(\tau_{A}\right)_{O}}{\Delta x_{A}{ }^{2}} \pi R_{p}^{2}$. Therefore, the linewidth is expressed as:

$$
d=2 y_{D O}=2 \sqrt{2} R_{S} \sqrt{\log \left(\frac{I_{0 L} \tau_{L}}{\sqrt{2 \pi} D_{O} R_{S} \Delta x_{L}}\right)}
$$

We have used equation (6) as a model function to fit the experimental linewidths as a function of beam current (Figure 5c) with $D_{O}$ and $R_{s}$ as fit parameters. All three data sets were fitted simultaneously with $R_{s}$ as a common/shared parameter. Onset doses determined from the fit follow the same trend as experimental ones. The observed systematic mismatch can be attributed to the fact that we excluded the likely $R_{s}$ dependence on material and, as discussed, contributions from primary beam and other sources ${ }^{29}$.

The analysis of the experimental data and the model both suggest that a precursor with smaller molecular weight combined with a decrease in the line dose could produce sub- $4 \mathrm{~nm}$ features. Indeed, by decreasing the beam current down to (0.21 pA) we patterned 3-nm lines on octane (see Supporting Information, Figure S5), which were, however, fragmented. This result indicates that the practical feature size limit is defined by stochastic effects ${ }^{30}$. By lowering the beam current we are decreasing the number of crosslinking events, but 
in doing so we also enhance statistical fluctuations along the patterned line. These fluctuations result in line roughness and fragmentation and are the main factor controlling the ultimate feature size limit.

In conclusion, OIRL is a new approach to nanopatterning that works with small organic molecules, which are difficult to be accessed by other techniques. After e-beam irradiation, molecules within the exposed areas are crosslinked and their solid/vapor phase transition temperatures raises. Therefore, OIRL does not require chemical development and allows the removal of the unexposed molecules by heating. The technique has no limitation on what kind of substrates or functional substrates it can handle, and can be cycled in-situ to achieve direct 3D nanopatterning.

Aiming at achieving full control over the result of the exposure process in view of tuning its characteristics and properties, in this work we have taken a step towards understanding the physical and chemical processes occurring when OIRs are irradiated by an electron beam. To establish a link between resist characteristics and outcome of the OIRL process, we have patterned linear hydrocarbons of varying molecular weight. Our results show that OIR precursors with lower molecular weight produce smaller features under the same patterning conditions. We have successfully fabricated small continuous features down to $4.5 \mathrm{~nm}$, and, more importantly, learned how to control the feature size by choosing a specific combination of beam and material parameters. With fixed beam parameters, the pattern size is primarily controlled by the onset dose, which we found to be inversely proportional to the precursor molecular weight. This trend, observed experimentally in polymer-based resists with much larger molecular weight, confirms that organic ices behave as regular negative tone resists, ensuring full compatibility of OIRL with existing lithographic protocols, while also opening new fields of application. Currently, we are focusing our efforts towards the use of OIRL in nanoscale additive manufacturing of functional materials, and biocompatible hydrogels for tissue engineering and soft robotics. 
a
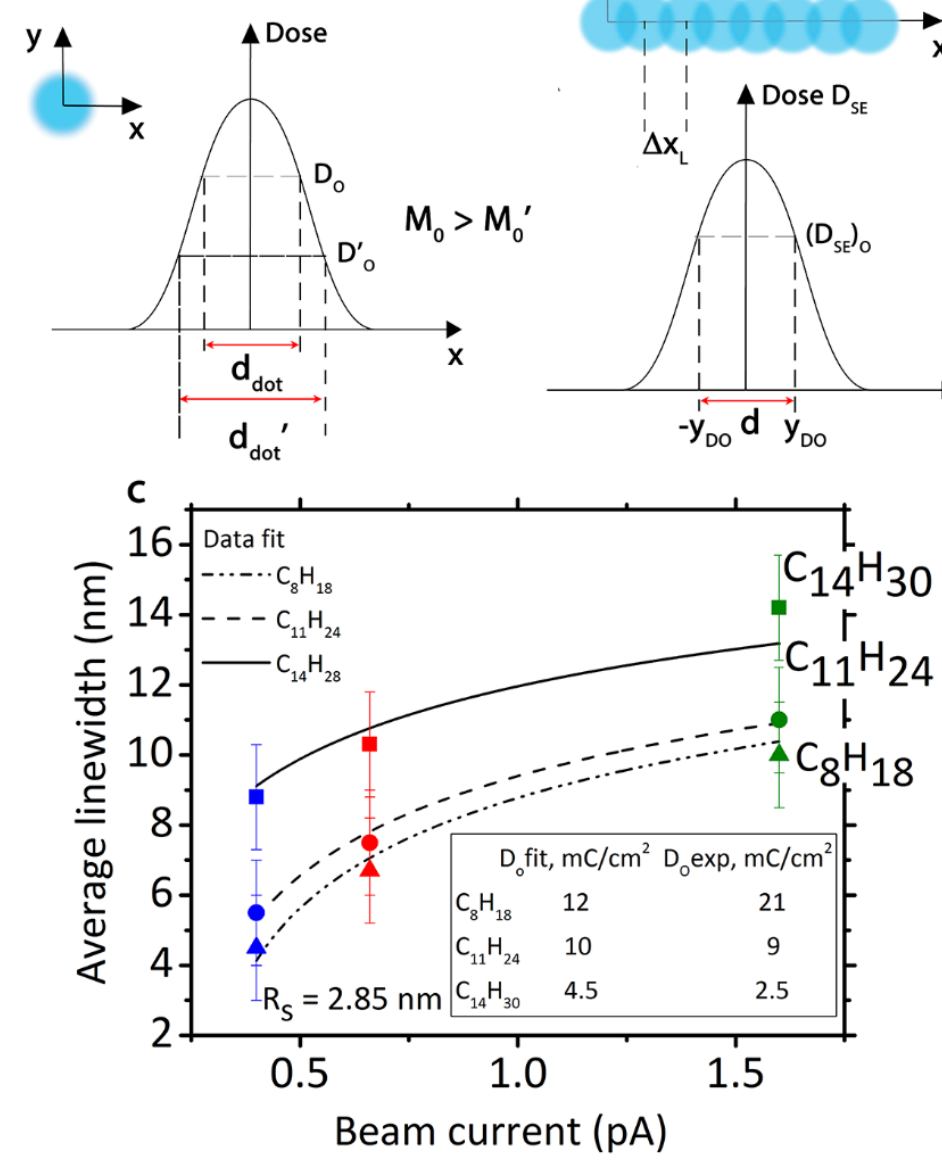

Figure 5. (a) Effect of Gaussian beam shape and the onset doses $D_{0}$ and $D_{\mathrm{o}}$ ' on the resulting isolated feature sizes $d_{\mathrm{dot}}$ and $d_{\mathrm{dot}}$, respectively. (b) Effect of secondary electrons Gaussian beam shape and contribution from the exposure of neighboring pixels on the resulting line feature size. (c) Experimental values of linewidths as a function beam current $/ 0$ fitted to Equation (6) with values of onset doses as parameters. Three datasets were fitted simultaneously with $R_{\mathrm{s}}$ as common parameter. 


\section{Author information}

\section{Corresponding author}

Email: marco.beleggia@cen.dtu.dk

\section{Author contributions}

The experiments were designed by A.E., A.H. and M.B., and carried out by A.E.; modelling and data analysis were performed by A.E. and M.B.; A.E. drafted the manuscript; A.E. and D.Z. created the illustrations; all the authors discussed the results and contributed to writing and revisions of the manuscript.

Notes

The authors declare no competing financial interests.

\section{Acknowledgments}

A.E. and M.B. acknowledge financial support from the VILLUM foundation. D.Z. acknowledges funding from the EU Horizon 2020 Marie Skłodowska-Curie Actions, H.C. Ørsted COFUND, grant agreement No. 713683.

\section{References}

(1) Pi, S.; Ghadiri-Sadrabadi, M.; Bardin, J. C.; Xia, Q. Nat. Commun. 2015, 6 (1), 7519.

(2) Ladd, T. D.; Jelezko, F.; Laflamme, R.; Nakamura, Y.; Monroe, C.; O’Brien, J. L. Nature 2010, 464 (7285), 45-53.

(3) Jahani, S.; Jacob, Z. Nat. Nanotechnol. 2016, 11 (1), 23-36.

(4) Geim, A. K.; Grigorieva, I. V. V Nature 2013, 499 (7459), 419-425.

(5) Aspelmeyer, M.; Kippenberg, T. J.; Marquardt, F. Rev. Mod. Phys. 2014, 86 (4), 1391-1452. 
(6) Sparreboom, W.; van den Berg, A.; Eijkel, J. C. T. Nat. Nanotechnol. 2009, 4 (11), 713-720.

(7) Faia-Torres, A. B.; Goren, T.; Textor, M.; Pla-Roca, M. Comprehensive Biomaterials; Elsevier, 2011, 181-201.

(8) Christman, K. L.; Enriquez-Rios, V. D.; Maynard, H. D. Soft Matter 2006, 2 (11), 928.

(9) Nie, Z.; Kumacheva, E. Nat. Mater. 2008, 7 (4), 277-290.

(10) Bat, E.; Lee, J.; Lau, U. Y.; Maynard, H. D. Nat. Commun. 2015, 6 (1), 6654.

(11) von Freymann, G.; Ledermann, A.; Thiel, M.; Staude, I.; Essig, S.; Busch, K.; Wegener, M. T Adv. Funct. Mater. 2010, 20 (7), 1038-1052.

(12) Gangnaik, A. S.; Georgiev, Y. M.; Holmes, J. D. Chem. Mater. 2017, 29 (5), 1898-1917.

(13) Hagen, C. W. Appl. Phys. A 2014, 117 (4), 1599-1605.

(14) Han, A.; Vlassarev, D.; Wang, J.; Golovchenko, J. A.; Branton, D. Nano Lett. 2010, 10 (12), 50565059.

(15) Tiddi, W.; Elsukova, A.; Le, H. T.; Liu, P.; Beleggia, M.; Han, A. Nano Lett. 2017, 17 (12), 7886-7891.

(16) Tiddi, W.; Elsukova, A.; Beleggia, M.; Han, A. Microelectron. Eng. 2018, 192, 38-43.

(17) Hong, Y.; Zhao, D.; Liu, D.; Ma, B.; Yao, G.; Li, Q.; Han, A.; Qiu, M. Nano Lett. 2018, 18(8), 5036-5041.

(18) Hu, X.; Wang, H.; Zhai, C.; Ge, H.; Cui, Y. J. Mater. Chem. C 2016, 4(47), 11104-11109.

(19) Mølgaard Mortensen, P.; Willum Hansen, T.; Birkedal Wagner, J.; Degn Jensen, A. Ultramicroscopy 2015, 152, 1-9.

(20) Malis, T.; Cheng, S. C.; Egerton, R. F. J. Electron Micr. Tech. 1988, 8 (2), 193-200.

(21) Manako, S.; Fujita, J.; Ochiai, Y.; Nomura, E.; Matsui, S. Jpn. J. Appl. Phys. 1997, 36 (Part 2, No. 6A), L724-L726. 
(22) Dey, R. K.; Cui, B. Nanotechnology 2013, 24 (24), 245302.

(23) Grigorescu, A. E.; Hagen, C. W. Nanotechnology 2009, 20 (29), 292001.

(24) Chen, Y. Microelectron. Eng. 2015, 135, 57-72.

(25) Mandelkern, L.; Prasad, A.; Alamo, R. G.; Stack, G. M. Macromolecules 1990, 23 (15), 3696-3700.

(26) Reusch, W. H. An Introduction to Organic Chemistry; Holden-Day, 1977.

(27) Jabbarzadeh, A.; Atkinson, J. D.; Tanner, R. I. Macromolecules 2003, 36(13), 5020-5031

(28) Egerton, R. F. Electron Energy-Loss Spectroscopy in the Electron Microscope; Springer, 2011.

(29) Manfrinato, V. R.; Wen, J.; Zhang, L.; Yang, Y.; Hobbs, R. G.; Baker, B.; Su, D.; Zakharov, D.; Zaluzec, N. J.; Miller, D. J.; Stach, E. A.; Berggren, K. K. Nano Lett. 2014, 14(8) 4406-4412

(30) Ocola, L. E.; Stein, A. J. Vac. Sci. Technol. B. 2006, 24 (6), 3061.

Supporting Information Available: description of the experimental procedures and guidelines for patterning organic ice resists in the TEM; patterning 3-nm lines on octane; width estimation of the patterned lines from bright-field TEM images; onset dose, contrast and saturation dose in OIRs and polystyrene resists with different molecular weights. 